\title{
Capacity gain with an alternative LTE railway communication network
}

\author{
Sniady, Aleksander; Soler, José
}

Published in:

Proceedings of the 7th International Workshop on Communication Technologies for Vehicles, Nets4Cars

Publication date:

2014

Link back to DTU Orbit

Citation $(A P A)$ :

Sniady, A., \& Soler, J. (2014). Capacity gain with an alternative LTE railway communication network. In Proceedings of the 7th International Workshop on Communication Technologies for Vehicles, Nets4Cars IEEE.

\section{General rights}

Copyright and moral rights for the publications made accessible in the public portal are retained by the authors and/or other copyright owners and it is a condition of accessing publications that users recognise and abide by the legal requirements associated with these rights.

- Users may download and print one copy of any publication from the public portal for the purpose of private study or research.

- You may not further distribute the material or use it for any profit-making activity or commercial gain

- You may freely distribute the URL identifying the publication in the public portal

If you believe that this document breaches copyright please contact us providing details, and we will remove access to the work immediately and investigate your claim 


\title{
Capacity gain with an alternative LTE railway communication network
}

\author{
Aleksander Sniady and José Soler, IEEE members \\ Networks Technology and Service Platforms \\ DTU Fotonik \\ Technical University of Denmark \\ Kgs. Lyngby, Denmark \\ \{alesn, joss $\} @$ fotonik.dtu.dk
}

\begin{abstract}
GSM-Railways (GSM-R) is the first international communication network designed specifically for railways. Most of the deployed GSM-R networks were initially used only to provide the railway voice communication. However, as more of these GSM-R networks begin to support the European Train Control System (ETCS) signaling, the capacity of GSM-R turns out to be insufficient. GSM-R cannot fulfill the railway requirements, in terms of the number of simultaneous ETCS connections. This is why, alternative, more efficient communication technologies should be considered by railways, such as 3GPP Long Term Evolution (LTE). This paper describes how to adapt the reversible multi-chain/channel queuing system to model an LTE cell serving ETCS-equipped trains. It is proposed to use the multiple user chains available in the model to represent varying bitrate in LTE radio access network. Using this model, LTE and GSM-R are compared in terms of capacity on an example at Copenhagen Main Train Station. The purpose of this work is to demonstrate the benefits that railway operators and infrastructure managers can expect from the introduction of LTE, as a telecommunication technology for railway control signaling and additional applications.
\end{abstract}

Keywords-GSM-R; LTE; ETCS; ERTMS; railway signaling; mobile communciation

\section{INTRODUCTION}

Although the popularity of GSM-R networks is still growing in Europe and other countries around the world [1], GSM-R technology already exposed its various shortcomings. These shortcomings have been broadly described in scientific literature, as well as technical reports: [2], [3], [4], [5].

The most critical problem with GSM-R is its insufficient capacity, i.e. insufficient number of transmission channels. GSM-R has to provide capacity for internal railway voice communication and ETCS signaling. However, there may not be enough GSM-R channels to provide the required ETCS connectivity to all the trains in some high concentration railway locations such as main train stations. For instance, such a situation occurs at Copenhagen Main Train Station, as described further in this paper. This implies that the number of GSM-R channels may become the element limiting the number of trains operating in a given area [2]. Desirably, the only limitation should be related to the capacity of the railway infrastructure and not the capacity of the communication infrastructure.

Due to this capacity limitation, GSM-R has to be eventually replaced by an alternative communication technology. LTE is a likely candidate to replace GSM-R in the future for various reasons. First of all, LTE has a number of advantages over GSM (and derivatives such as GSM-R) in terms of capacity and capabilities. LTE is better suited for data communication, because it is a fully packet-switched based network. Moreover, since LTE is developed by 3GPP, it provides standardized interworking mechanisms with GSM. Thanks to these mechanisms, migration from GSM-R to LTE is easier than migration to some other technology. Finally, LTE (and LTE-A) is the latest family of 3GPP standards. Thus, it has much lower obsolescence risk than earlier standards, such as UMTS.

LTE advantages over GSM are well known in the telecommunication industry [6]. Despite that, LTE has to be analyzed explicitly in a railway environment. This has to be done in order to demonstrate to railway operators and infrastructure managers, how LTE can solve their specific communication issues. Moreover, it must be proven that LTE is able to fulfill safety requirements set by the railway industry. Hence, a growing number of research works investigate LTE as a likely future communication technology for railways and other vehicles [7], [8], [9].

The purpose of this paper is to explicitly demonstrate the capacity improvements that can be expected from replacing GSM-R with LTE. The analytical model, used for this demonstration, is supposed to show whether LTE offers sufficient capacity for ETCS signaling at a major train station.

The paper is organized as follows. The next section presents ETCS capacity requirements based on a specific example of Copenhagen Main Train Station. In section III, the communication capacity of a GSM-R cell is calculated. Then, in section IV, an LTE cell capacity is calculated using a new approach to the reversible multi-chain/channel queuing system. Finally, section V compares GSM-R and LTE capacities with the Copenhagen Main Train Station requirements and concludes the paper.

\section{ETCS COMMUNICATION CAPACITY REQUIREMENTS AT COPENHAGEN MAIN TRAIN STATION}

ETCS is a railway command-control system, ensuring safe and efficient train movement [10]. For the purposes of ETCS, the underlying railway communication network needs to provide connectivity between a Radio Block Controller (RBC) and On-Board Units (OBUs) in trains. The RBC is an ETCS server supervising train movement. OBU is an ETCS element located in every train locomotive. OBUs are connected over a mobile radio network, while $\mathrm{RBC}$ is connected via a fixed wired network. RBC and OBUs exchange various messages in order to control trains. For instance, OBUs transmit Location Update messages informing the $\mathrm{RBC}$ about their current train location. On the other hand, the RBC sends Movement Authorities to OBUs allowing trains to continue driving for a specific distance [10].

The supporting railway communication network has to offer sufficient capacity to provide ETCS connectivity between $\mathrm{RBC}$ and each OBU (i.e. each train). The necessary number of 
ETCS connections varies in different railway locations, depending on the expected number of trains. This work uses an example of Copenhagen Main Train Station, which is the biggest train station in Denmark, with the highest number of trains arriving and departing per hour. As a consequence, it is the location with the highest demand for ETCS connections [2]. Moreover, it is recommended that handovers between cells should be avoided at locations where low transmission delay is required, such as train stations [11], [12]. Thus, single-cell coverage is considered for the whole train station. According to calculations presented in [13], a single cell of the mobile network at Copenhagen Main Train Station has to support 27 concurrent ETCS connections. In the future this requirement is expected to grow to about 40 concurrent ETCS connections.

\section{GSM-R CELL RADIO LINK CAPACITY ESTIMATION}

In GSM-R, which is a circuit-switched based network, each OBU has a dedicated bidirectional circuit connection established in the network in order to transmit ETCS signaling or voice communication. This circuit is reserved for the particular OBU. Thus, network resources, both on the radio link as well as in the backbone network, are reserved for a single OBU-RBC connection. The ETCS connection cannot be shut down when no messages are transmitted, because a connection reestablishment would consume too much time. As a result, network resources (i.e. circuits) are reserved continuously, even in the absence of communication. On the other hand, the circuit connection provides a stable transmission performance in terms of bandwidth, delay and packet loss.

ETCS transmission pattern is not important in GSM-R. As explained, this is because, a GSM-R connection always occupies one fixed user transmission channel on the radio link. The transmission channel cannot be shared with other connections even if the channel is underutilized (e.g. due to a transmission pattern with very infrequent messages). All in all, in order to provide sufficient capacity for ETCS signaling, a GSM-R network should provide one user traffic channel for each train (OBU).

Note that, for every GSM-R channel in downlink there is always a corresponding channel in uplink. Moreover, user is always assigned in a bundle of uplink and downlink channels. Thus, all the considerations concerning GSM-R channels apply equally to uplink and downlink directions.

In Europe, GSM-R operates in a dedicated frequency band of $4 \mathrm{MHz}$ (4 MHz for uplink and $4 \mathrm{MHz}$ for downlink) [10]. $200 \mathrm{kHz}$ are consumed by guard bands, which prevent interference from the neighboring frequency bands used by other technologies. In the remaining $3.8 \mathrm{MHz}$, it is possible to place 19 frequency channels of $200 \mathrm{kHz}$ each. The channels are numbered from 955 to 973 .

If a frequency channel is used in one GSM-R cell then it cannot be reused in the neighboring cells to prevent interference. Thus, not all of the frequency channels can be used in a single cell. In practice, usually seven unique frequency channels are required in order to provide GSM-R coverage over a wide area [12], [14]. Thus, the available 19 frequency channels are divided into seven sets with dedicated frequencies. As a result, a cell can use only one of the sets, so it can use two or three frequency channels. This is shown in Table 1, which contains an exemplary channel distribution based on UIC GSM-R Procurement Guide [14].

Each frequency channel is shared between users using Time Division Multiple Access (TDMA). There are 8 time-slots in
TABLE 1. EXEMPLARY DIVISION OF GSM-R FREQUENCY CHANNELS INTO 7 SETS. BASED ON [14]

\begin{tabular}{|c|l|c|c|}
\hline Set & $\begin{array}{c}|c| \\
\text { Frequency } \\
\text { channels }\end{array}$ & $\begin{array}{c}\text { Number of user } \\
\text { traffic channels }\end{array}$ & $\begin{array}{c}\text { Number of } \\
\text { signaling channels }\end{array}$ \\
\hline $\mathrm{A}$ & $\mathrm{n}=955,962,969$ & 22 or 23 & 1 or 2 \\
\hline $\mathrm{B}$ & $\mathrm{n}=956,963,970$ & 22 or 23 & 1 or 2 \\
\hline $\mathrm{C}$ & $\mathrm{n}=957,964,971$ & 22 or 23 & 1 or 2 \\
\hline $\mathrm{D}$ & $\mathrm{n}=958,965,972$ & 22 or 23 & 1 or 2 \\
\hline $\mathrm{E}$ & $\mathrm{n}=959,966,973$ & 22 or 23 & 1 or 2 \\
\hline $\mathrm{F}$ & $\mathrm{n}=960,967$ & 15 & 1 \\
\hline $\mathrm{G}$ & $\mathrm{n}=961,968$ & 15 & 1 \\
\hline
\end{tabular}

each channel. Not all of them are used for user traffic, because in each cell one or two time-slots need to be reserved for GSM-R signaling. Hence, in the best case (with the highest capacity) a GSM-R cell has 3 frequency channels, i.e. 24 timeslots, where one of these time-slots is reserved for signaling. This implies that a GSM-R cell can have at most 23 user-traffic channels, as presented in the second column of Table 1 .

\section{LTE CELL RADIO LINK CAPACITY ESTIMATION}

In LTE, which is a packet-switched based network, the transmission resources are shared between users (OBUs). Thus, there is no hard limit on the number of OBUs in an LTE cell. The maximum number of OBUs that an LTE cell can serve is defined indirectly by the transmission performance. New OBUs can be accepted as long as the ETCS transmission performance fulfills requirements e.g. in terms of delay and packets loss.

ETCS requirements for circuit-switched based networks are defined by UIC [8]. In case of packet-switched based networks these requirements are still being discussed and are not finalized. This is why, in this work, tentative requirements published by the Danish national railway infrastructure manager, Banedanmark are used [2]. These requirements concern parameters such as mean transfer delay of ETCS messages, ETCS data integrity, and received signal strength at OBU.

The following analysis is focused on the mean transfer delay of ETCS messages, because it is the critical parameter being the most dependent on the number of OBUs in a cell. This is because the more OBUs in the system, the bigger the offered ETCS traffic is. According to ETCS requirements, the mean transfer delay of a 128-byte ETCS message cannot exceed $0.5 \mathrm{~s}$ [2].

Furthermore, the analysis deals only with the LTE radio access, as it is the place where the network resources are scarce and cannot be easily extended due to the railway radio bandwidth being limited to $4 \mathrm{MHz}$. Thus, the radio access is the most probable place of a bottleneck in the network.

\section{A. ETCS tranmission pattern}

In contrast to GSM-R, in the case of LTE, the transmission pattern of ETCS messages has to be known. In the presented model model, it is assumed that each OBU sends an ETCS message to the $\mathrm{RBC}$ at time intervals following an exponential distribution with a mean value of $30 \mathrm{~s}$. Also the RBC sends an ETCS message to each of the OBUs at time intervals following an exponential distribution with a mean value of $30 \mathrm{~s}$. These time values are based on the assumption that a movement 
authority and a position update messages are transferred every half a minute. ETCS messages have a constant size of 128 bytes according to the size specified in the ETCS requirements [2]. Moreover, each ETCS message is acknowledged with a message of equal size. Thus, on average 4 ETCS messages are transmitted per minute in each direction (uplink and downlink) Based on this, the ETCS message arrival rate $\left(\lambda_{\text {ETCS }}\right)$, i.e. the arrival at the LTE interface, is as follows:

$$
\lambda_{\text {ETCS }}=\frac{4 \mathrm{msg}}{60 \mathrm{~s}}=\frac{1}{15} \frac{\mathrm{msg}}{\mathrm{s}}
$$

The ETCS message size ( $\left.m_{\text {ETCS }}\right)$, initially of 128 bytes, is additionally increased by 64 bytes to model the overhead due to IP, TCP and RLC protocol headers:

$$
m_{\text {ETCS }}=(128+64) \frac{\text { byte }}{m s g}=1536 \frac{\text { bit }}{\mathrm{msg}}
$$

\section{B. Model of LTE radio access}

In order to estimate the transfer delay over the LTE radio, we propose to use the $\sum_{j=1}^{N} M_{j} / G_{j} / n-G P S$ reversible multichain/channel queuing system model presented in [16]. The model represents a system with $n$ channels and an infinite queue. There are $\mathrm{N}$ types of messages (also referred to as chains) in the system. In the model, each arriving message requests one channel. If the channel demand is higher than the available number of channels, the channels are shared between the messages (Processor Sharing). The $\sum_{j=1}^{N} M_{j} / G_{j} / n-G P S$ reversible multi-chain/channel queuing system has been chosen to model the analyzed LTE cell, because the system includes multiple channels (used in this analysis for modeling LTE Resource Blocks) and multiple user chains (used in this analysis for modeling varying bitrates in LTE). Typically, the multiple user chains represent various applications transmitted over a network. The presented here approach proposes to use the user chains to represent varying bitrates of LTE radio. More explanations on how he model is used are presented in the following sections.

The state of the system $(\bar{x})$ is defined by the number of messages in the system:

$$
\bar{x}=\left(x_{1}, x_{2}, \ldots, x_{j}, \ldots, x_{N},\right)
$$

where $x_{j}$ is the number of messages of type $j$. A descriptive state diagram of the modeled system is shown in Figure 1. Note, that the diagram shows a case with only two message types. Thus, the states on the diagram are defined by the number of messages of type 1 and the number of messages of type $2: \bar{x}=\left(x_{1}, x_{2}\right)$.

The transitions between the states are displayed in the diagram. Each transition is described by its rate. A transition can occur either due to the arrival of a new message or due to a message departing from the system (i.e. completed transmission over the LTE radio access network).

Message arrivals to the system are independent of the state of the system. This is because, in reality the OBUs and the RBC transmit ETCS messages regardless of the number of messages being already in the communication system. This is shown in Figure 1, where the arrival rates for each message type are the same in all the states.

On the other hand, the departure of messages, in each state, is dependent on the state of the system, i.e. the number of messages in the system. In the states where the number of messages is smaller or equal to the number of available channels ( $\left.\sum \mathrm{x} \leq \mathrm{n}\right)$ each message gets its dedicated channel.

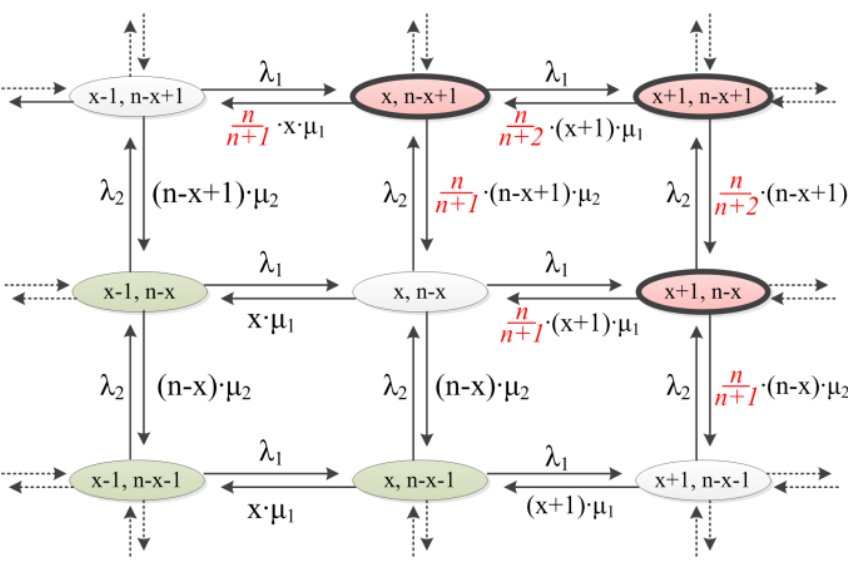

Figure 1. State transition diagram of the system in case with 2 message types. In the green states, there are less messages in the system than available channels $\left(\sum x<n\right)$. In the white states there are as many messages as channels $\left(\sum x=n\right)$, thus, every message has its own channel. In the pink states with a thick outline, there are more messages than available channels $\left(\sum x>n\right)$, thus, the messages have to share the available resources. This sharing is modeled by the reduction factors (marked with red font), which reduce the service rates proportionally to the number of messages in the system.

The more messages are in the system, the more channels are used. Therefore, more messages are being served simultaneously. As a result, more messages depart from the system per time unit. When there are more messages than available channels $\left(\sum x>n\right)$, the channels have to be shared between the messages. These states are marked with pink color and a thick outline in Figure 1. The channel sharing is used to model the situation when an arriving message has to wait for LTE radio access resources. In order to include resource sharing in the model, the departure transitions are scaled by a reduction factor, as explained further in [16]. In Figure 1, the reduction factors are marked with red and italic font.

The arrival rates $\lambda_{\mathrm{j}}$ and the service rates $\mu_{j}$ are described in detail in the following subsections.

\section{State probabilities}

The full explanation of the model and its mathematical derivation are described in [16]. Here, only a brief overview of the most important elements is presented.

Firstly, in order to find theoretical performance of the system, the relative state probabilities need to be found using the following recursive equations [16]:

$$
q(x)=\left\{\begin{array}{cc}
0 & x<0 \\
1 & x=0 \\
\sum_{j=1}^{N} q_{j}(x) & x=1,2, \ldots, \infty .
\end{array}\right.
$$

where the contributions from each message types $q_{j}(x)$ is:

$$
q_{j}(x)= \begin{cases}\frac{\lambda_{j} \cdot q(x-1)}{\mu_{j} \cdot x} & x \leq n \\ \frac{\lambda_{j} \cdot q(x-1)}{\mu_{j} \cdot n} & x>n\end{cases}
$$

In these equations it should be noted that the global state probability $q(x)$ is a sum of contributions from each message type $q_{j}(x)$. Moreover, $q_{j}(x)$ differs between the case when there are free channels $(x \leq n)$ and the case when channels have to be shared between the messages $(x>n)$. Finally, the 
system cannot be in a state with negative a number of messages. Hence, $q(x<0)=0$.

Then, in order to find the corresponding true state probabilities $p(x)$ and $p_{j}(x)$ the relative state probabilities need to be divided by a normalization factor:

$$
\begin{gathered}
Q=\sum_{x=0}^{\infty} q(x) \\
p(x)=\frac{q(x)}{Q} \\
p_{j}(x)=\frac{q_{j}(x)}{Q}
\end{gathered}
$$

After the normalization the sum of all probabilities equals to one $\left(\sum p(x)=1\right)$.

\section{Performance measures}

Based on the state probabilities it is possible to find the mean queue length $\bar{L}_{j}$ for the stream of type-j messages:

$$
\bar{L}_{j}=\sum_{x=0}^{\infty} x \cdot p_{j}(x)
$$

Subsequently, it is possible to find the mean sojourn time, which is a combined waiting and service time. It is the overall transmission time (sojourn time) that is of interest in this work. The mean sojourn time for type $\mathrm{j}$ messages is:

$$
\bar{W}_{j}=\frac{\bar{L}_{j}}{\lambda_{j}}
$$

And finally the average sojourn time for messages of all types, which is the final result expected form the calculations, becomes:

$$
\bar{W}=\sum_{j=1}^{N} \frac{\lambda_{j}}{\lambda} \cdot \bar{W}_{j}
$$

\section{E. Number of channels}

LTE can operate in $1.4,3,5,10,15$ or $20 \mathrm{MHz}$ bandwidth. Depending on the bandwidth, there is a different number of LTE Resource Blocks (RB) available in a cell, as shown in Table 2 [6].

TABLE 2. NumBER OF RESOURCES BLOCKS (RB) IN LTE BANDWIDTH

\begin{tabular}{|c|c|c|c|c|c|c|}
\hline Bandwidth, MHz & 1.4 & 3 & 5 & 10 & 15 & 20 \\
\hline RB & 6 & 15 & 25 & 50 & 75 & 100 \\
\hline
\end{tabular}

In order to make a direct comparison with GSM-R, which operates in $4 \mathrm{MHz}$ bandwidth, it is assumed that the modeled LTE cell also operates in $4 \mathrm{MHz}$ bandwidth. Due to that, a nonstandard number of RB is chosen: $20 \mathrm{RB}$. In the model, the RB are represented as channels, thus there are $n=20$ channels available.

\section{F. Message types}

Usually, in the reversible multi-chain/channel queuing system model, the different message types (different chains) represent different applications. In the presented here approach, these different message types represent different radio transmission bitrates of LTE. An OBU can experience various transmission bitrates depending on the Signal to Interference and Noise Ratio (SINR), as explained in [17]. Depending on its location, an OBU receives higher or lower bitrate. This is because, the further an OBU is from the eNodeB, the weaker the received signal is and the higher the interference from the neighboring cells is. As a result, SINR observed by the OBU decreases. Depending on SINR, each OBU chooses a Modulation and Coding Scheme (MCS). This MCS determines the OBU transmission bitrate. If the SINR is low, the more robust MCS (with lower modulation and more redundant coding scheme) has to be used. This implies that fewer bits are transmitted in a single RB.

In order to model this effect of varying bitrate, we propose to assign each OBU to one of the $\mathrm{N}$ message types of the previously described model. Thus, OBU belonging to type $\mathrm{j}$ transmits and receives messages of type $\mathrm{j}$. Each message type is characterized by the bitrate $b_{j}$. Every OBU produces ETCS messages with the same rate (arrival rate), but these messages are send slower or faster depending on the bitrate. Bitrate values are determined using tables 7.1.7.1-1, 7.1.7.2.1-1 and 8.6.1-1 from 3GPP standards [18]. The maximum bitrate in downlink is $712 \mathrm{Kbit} / \mathrm{s}$ per RB (in case of maximum MCS $=28$ ). Currently most LTE devices do not support 64QAM modulation in uplink (from OBU towards eNodeB). In order to model that, the uplink MCS is limited to 20, which results in a maximum uplink bitrate of $408 \mathrm{Kbit} / \mathrm{s}$ per RB. Additionally, the bitrates have been reduced by $30 \%$ in order to include the negative impact of the expected retransmissions over the radio access [6].

\section{G. Arrival and service rates}

The arrival rate of type $\mathrm{j}$ messages $\left(\lambda_{\mathrm{j}}\right)$ is proportional to the number of trains of type $\mathrm{j}$ (trains $\mathrm{t}_{\mathrm{j}}$ ) and proportional to the ETCS message arrival rate ( $\left.\lambda_{\text {ETCS }}\right)$ expressed in $\operatorname{~sec}^{-1}$ :

$$
\lambda_{j}=\text { trains }_{j} \cdot \lambda_{\text {ETCS }}
$$

The service rate of type $\mathrm{j}$ messages $\left(\mu_{j}\right)$ is proportional to the bitrate $\left(b_{j}\right)$ expressed in bits/s and inversely proportional to the ETCS message size $\left(m_{E T C S}\right)$ expressed in bits:

$$
\mu_{j}=\frac{b_{j}}{m_{E T C S}}
$$

Additionally, it is assumed that the message transmission cannot be shorter than $1 \mathrm{~ms}$. This is because, in LTE, an OBU gets transmission resources allocated for at least $1 \mathrm{~ms}$ [6].

\section{H. Calculation results}

Figure 2 shows the mean sojourn time results obtained from the presented model. The graph illustrates the impact of the number of trains (OBUs, UEs) in the LTE cell on the mean sojourn time of ETCS messages, at the LTE radio access. The analysis considers a very wide, unrealistic, range of trains (up to 80000) in order to find the limits where ETCS traffic actually overloads the LTE radio-cell capacity.

Looking at the uplink plot in Figure 2, it can be seen that the mean sojourn time is barely affected by the increasing number of trains until the number of trains reaches 40000 . In this range, the mean sojourn time grows from $5.9 \mathrm{~ms}$ (100 trains) to $6.2 \mathrm{~ms}$ (40000 trains). Only then, the mean sojourn time begins to rise more noticeably. When the number of trains exceeds 52000, the uplink capacity is surpassed.

Similarly, in the downlink case, the mean sojourn time firstly grows very slightly from $4.3 \mathrm{~ms}$ (100 trains) to $4.7 \mathrm{~ms}$ (60000) trains. Then, above 60000 trains, it begins to rise more rapidly and when the number of trains reaches 72000 , the downlink capacity is exceeded. 


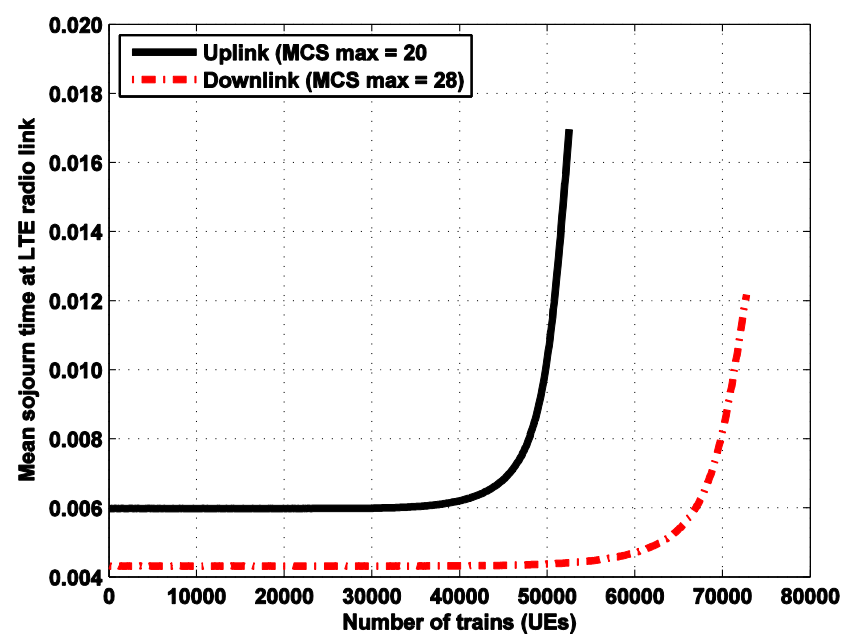

Figure 2. The relation between the number of ETCS-equipped trains (UEs, OBUs) in the modeled LTE cell and the mean sojourn time over the LTE radio link.

Uplink performance is the factor limiting the possible number of trains served in a single cell. This is because the uplink mean sojourn time is higher than the downlink one. Besides, the uplink capacity can accommodate fewer trains, as shown in Figure 2.

The mean transfer delays obtained from the model are in the range of few milliseconds. Thus, the values are a few orders of magnitude smaller than the ETCS requirement of $0.5 \mathrm{~s}$. It has to be stressed that this analysis only focused on the radio link, so an additional delay will be introduced by other elements and layers in the network. Despite that, the results show that ETCS signaling generates traffic that is significantly smaller than the available LTE radio capacity. In a realistic scenario, where there are e.g. 40 trains in a cell, ETCS signaling on its own cannot exhaust the available LTE capacity. Hence, railway LTE network can be used for much more than only ETCS signaling. Thanks to the available LTE capacity, railway operators could introduce a variety of new applications, such as maintenance data exchange, remote software updates or video surveillance. These services cannot be provided in GSM network.

\section{CONCLUSIONS}

ETCS is an essential railway communication-based service. Every train in an ETCS-based railway system must be connected to the RBC. Thus, the railway communication network needs to provide sufficient capacity to enable this ETCS connectivity. As it was estimated in the paper, based on railway technical reports, Copenhagen Main Train Station requires currently 27 simultaneous ETCS connections in a single mobile cell. What is more, in the future this requirement is going to grow to about 40 connections.

GSM-R technology, which is currently being deployed in Denmark, does not provide sufficient capacity to support all these connections. As it was presented in the paper, a GSM-R radio-cell provides capacity for approximately 23 ETCS connections per cell. Furthermore, as the connection demand is growing, this problem of insufficient GSM-R capacity is going to worsen.

As it is shown in the paper, an alternative LTE-based railway communication network offers capacity greatly exceeding ETCS requirements. LTE can support ETCS connectivity for many more trains than can be ever expected at Copenhagen Main Train Station. Thus, the additional capacity allows for various new communication-based railway applications.

Furthermore, the transfer delay over an LTE radio access is very low. It is significantly lower than the upper delay limit set by the ETCS requirements at $0.5 \mathrm{~s}$. These conclusions about LTE performance are based on the theoretical calculation results from the reversible multi-chain/channel queuing system model. In this paper, this queuing system was applied in an innovative way for the purpose of modeling an LTE radio-cell.

In summary, the presented analysis shows that LTE can solve the insufficient capacity issue of GSM-R. Therefore, LTE should be considered as a viable alternative to GSM-R.

\section{ACKNOWLEDGMENTS}

This paper is supported by the Danish Council for Strategic Research through the RobustRailS project, strengthening the railway research in Denmark:

http://www.robustrails.man.dtu.dk/

\section{REFERENCES}

[1] UIC, "ERTMS Atlas 2012", 10th UIC ERTMS World Conference in Stockholm, April 2012.

[2] D. G. Fisher, "Requirements on the GSM-R Network for ETCS Support", Technical report, Banedanmark, 2008.

[3] S. F. Ruesche, J. Steuer and K. Jobmann, "The European Switch. Packet-Switched Approach to a Train Control System”, IEEE Vehicular Technology Magazine, pp. 37-46, September 2008.

[4] P. Sondi, M. Berbineau, M. Kassab and G. Mariano, "Generating Test Scenarios Based on Real-World Traces for ERTMS Telecommunication Subsystem Evaluation", Communication Technologies for Vehicles: $5^{\text {th }}$ International Workshop, Nets4Cars/Nets4Trains, Lecture Notes in Computer Science, Vol. 7865, pp. 223-231, Springer, 2013.

[5] A. Sniady and J. Soler, "An overview of GSM-R technology and its shortcomings", Proceedings of The $12^{\text {th }}$ International Conference on ITST, pp. 626-629, IEEE, 2012.

[6] M. Sauter, "From GSM to LTE: an introduction to mobile networks and mobile broadband", John Wiley \& Sons, 2011.

[7] J. Calle-Sánchez, et al., "Long Term Evolution in High Speed Railway Environments: Feasibility and Challenges", Bell Labs Technical Journal, Alcatel-Lucent, Volume 18, Issue 2, pp. 237-253, John Wiley \& Sons, 2013.

[8] A. Diaz Zayas, C. A. Garcia Perez and P. M. Gomez, "Third-Generation Partnership Project Standards: For Delivery of Critical Communications for Railways", IEEE Vehicular Technology Magazine, Vol. 9, Issue 2, pp. 58 - 68, June 2014.

[9] A. Vinel, "3GPP LTE Versus IEEE 802.11p/WAVE: Which Technology is Able to Support Cooperative Vehicular Safety Applications?", IEEE Wireless Communications Letters, Vol. 1, No. 2, pp. 125-128, April 2012.

[10] P. Winter, et al., “Compendium on ERTMS”, Eurail Press, 2009.

[11] ERTMS, "SUBSET-093: GSM-R Interfaces; Class 1 requirements", 2005

[12] J. Binningsbø, R. Baldersheim, J. P. Lauvstad, "GSM-R Radio Planning Guidelines", Technical report, Dok.nr.: 3A-GSM-038, Jernbaneverket Utbygging, 2006.

[13] A. Sniady and J. Soler, "Impact of the traffic load on performance of an alternative LTE railway communication network", Proceedings of The $13^{\text {th }}$ International Conference on ITS Telecommunications, pp. 396-401, IEEE, 2013.

[14] UIC, “GSM-R Procurement Guide”, ISBN: 2-7461-1315-5, Paris 2007.

[15] H. E. Jørgensen, "Kapacitetsforbedringsplan. Overbelastet infrastruktur." (In Danish), Technical report, Banedanmark, 2011.

[16] V. B. Iversen, "The Internet Erlang formula", Proc. NEW2AN/ruSMART, Lecture Notes in Computer Science, Vol. 7469, pp. 328-337, Springer, 2012

[17] H. Holma and A. Toskala, "LTE for UMTS - OFDMA and SC-FDMA Based Radio Access", John Wiley \& Sons, 2009.

[18] 3GPP, "TS 36.213: Evolved Universal Terrestrial Radio Access (E-UTRA); Physical layer procedures.", V11.3.0, 2013. 